\title{
El papel de la cintura escapular en la postura y el movimiento
}

\section{Resumen}

El propósito de este artículo es revisar la importancia de las funciones motoras de la cintura escapular en el desarrollo de la postura y el movimiento sin desconocer todos los factores que intervienen y son esenciales en el aprendizaje motor del niño. Esta interpretación, mira en detalle los eventos en común de los segmentos corporales que la conforman y sus patrones de movimiento en relación con la cintura escapular, con una intención: ser usados en la intervención. La revisión de esta información y su entendimiento puede ser usada como referencia para la evaluación y tratamiento en niños con desarrollo motor atipico y retraso motor.

Finalmente, se muestra la estructura escapular como un segmento corporal que juega un papel importante en múltiples funciones del cuerpo del niño durante su desarrollo y por tanto, la hace de interés interdisciplinario.

Desarrọllo Motor, Motricidad Fina, Motricidad Gruesa, Habilidad Motora Funcional, Quinesiología.
FT. Marlen Rojas Rojas

Fisioterapeuta. Especialista en Neurodesarrollo,

Asociación Norteamericana de Neurodesarrollo.

Especialista en Docencia Universitaria, Universidad del Rosario.

Docente del programa de Fisioterapia

de la Universidad del Rosario.

Email:marrojas@urosario.edu.co

\section{Abstrac}

The purpose of this article is to make a review on the importance of motor skills of shoulder girdle in movement development and postural control, without ignoring all factors that participate and are essential in child motor learning. This interpretation, observe in detail all com- 
mon factors of body structures which take part and its movement patterns related to shoulder girdle, one main intention: be used for intervention. Reviewing this information and its understanding can be used as a reference for assessment and treatment of abnormal development and motor delay.

Finally, it shows scapular structure as a body segment which plays an important role in multiple activities of the child's body during development and thus, makes it an interdisciplinary issue.

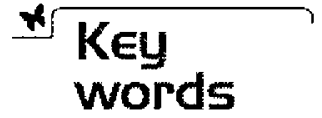

Motor Development, Fine Motor, Gross Motor, Functional Motor skills, Kinesiology.

\section{Introducción}

Se encuentran muchas referencias que hablan del desarrollo neuromotor del niño en su primer año de vida, pero, es poco frecuente que su interpretación aporte una intención que favorezca la comprensión de los procesos por los cuales una función motora se desarrolla a partir de otra y se hace dificil dirigir ese conocimiento a comprender el ¿porque hacer? ó ¿como construir? de cierta forma una intervención terapéutica.

Los terapeutas recalcan que la mayoria de los procesos de aprendizaje dependen de la habilidad para moverse. Por este motivo, la función motora se trabaja y se desarrolla con técnicas neurofisiológicas. Una vez conseguida la habilidad necesaria para moverse será cuando se comience con el aprendizaje de los autocuidados, actividades escolares, juegos y hobbies (1). Sin embargo, es importante tener en cuenta que un niño no se mueve únicamente gracias a la neurofisiología. La mente del niño almacena información desde todos sus sistemas sensoriales y, mediante los movimientos, el niño empieza a estructurar conclusiones con respecto al mundo espacial que lo rodea. Asi, el niño consigue utilizar sus funciones motoras en la mayoría de las actividades dia- 
rias, aprendiendo a responder a las demandas del medio.

De cualquier manera, y para responder a la necesidad planteada anteriormente, este artículo presenta una interpretación quinesiológica diferente sobre el trabajo de ese sistema flotante e influyente que es la cintura escapular.

La cintura escapular tiene relación directa e indirecta con casi todos los demás segmentos corporales: la cabeza y el cuello, el tronco, la reja costal, los miembros superiores, y la cintura pélvica por nombrar los mas directos. Además, interviene en funciones importantes como la respiración, la fonación, la motricidad fina, la adquisición de la posición sedente, y su paso a la posiciones sedente lateral y cuadrúpeda. La cintura escapular también, controla en parte la postura y el movimiento durante el gateo, el proceso de traslado a la posición de rodillas y a la posición bípeda.

Entender claramente el desarrollo de los movimientos en el complejo escápulo-humero-costoesternal y la función de sus múltiples relaciones, le facilita al terapeuta detectar vacios motores durante la evaluación del niño con situaciones neuromotoras especiales y organizar una intervención mas eficaz, ofreciendo los elementos de postura y movimiento necesarios para construir la función que se busca desde su disciplina: Motricidad fina, fonación, motricidad gruesa, respiración, etc.

Por ejemplo, es frecuente encontrar el niño cuya actitud postural le lleva a sentarse con la escápula en adducción y rotación interna, el hombro en hiperextensión, adducción y rotación interna y los codos en flexión limitando seriamente todo alcance y agarre con sus extremidades superiores. Conocer que esta actitud postural la presenta el niño en compensación a una extensión toráxica disminuida ó ausente dirige fácilmente al terapeuta a evaluar el trabajo del tronco superior y analizar que su intervención debe enfocarla a ganar la extensión motora funcional del tronco superior para que sus extremidades superiores se liberen en el patrón de movimiento antagónico, sólo entonces podrá el niño ser capaz de iniciar un alcance.

\section{Generalidades}

Es necesario revisar en forma general el desarrollo de los diferentes patrones de movimiento en el niño, para ubicar en este contexto la cintura escapular. También, es pertinente recordar los procesos que llevan a la estabilidad, el balance de la actividad motora y el soporte biomecánico para desarrollar una habilidad.

Para empezar a construir una habilidad, cada movimiento necesita una base estable que proporcione el apoyo para su ejecución; en el recién na- 
cido la flexión fisiológica proporciona esa base que aunque involuntaria da origen a los primeros movimientos voluntarios rotacionales en el plano extensor visto en los intentos de levantar la cabeza de manera asimétrica alrededor del primer mes y culminando con la extensión simétrica de la cabeza; este orden se repite en cada segmento corporal hasta lograr una extensión completa de tronco y extremidades en prono alrededor del sexto mes (2).

La flexión como movimiento voluntario emerge a partir de cada segmento que ha controlado la extensión y ahora le sirve de apoyo para que se desarrolle, al cuarto mes vemos buena parte a nivel de cabeza, al quinto, sexto se inicia en tronco y hacia el año se puede ver presente y funcional la flexión en tronco y pelvis con buen trabajo de abdominales rectos y oblicuos demostrado en el buen control de la transición de supino a sedente en el plano antero-posterior.
Posteriormente, el segmento corporal desarrollado en el control de flexión y extensión puede mostrar las rotaciones quienes no son más que el juego antagónico de grupos musculares ubicados en el mismo hemicuerpo. Es decir, en una rotación a la derecha resulta del control de una flexión anterior con una extensión posterior (3).

Motivado por los alcances y su curiosidad por desplazarse el niño necesita sumar los traslados de peso a las anteriores combinaciones y dominios, y así es llevado a controlar otro componente esencial en el movimiento y es la inclinación, cuya presencia desarrolla una elongación (contracción excéntrica) del lado que soporta peso con un acortamiento (contracción concéntrica) del lado que libera peso para desplazarse o levantarse (FIGURA 1). Este mecanismo es importante de tener en cuenta, pues de no presentarse el niño se cae hacia el lado que no soporta peso y la función se ve imposibilitada.

\section{Estabilidad dinámica y estática}

Analizar en forma global el desarrollo del movimiento hace necesario revisar también los conceptos de estabilidad que puede ser estabilidad estática o estabilidad dinámica. Partiendo del concepto básico del punto de apoyo como elemento indispensable para el movimiento "dame un punto de apoyo y moveré el mundo" (Atlas). Este apoyo es el segmento o punto que nos da la estabilidad y sin el cual no se po- dría dar un movimiento coordinado dirigido e intencionado como es el movimiento funcional.

Si observamos, cada vez que se presenta un movimiento, se tiene un segmento muy cercano que da la estabilidad, como es el caso de la elevación del brazo durante un alcance donde la escápula estabiliza el hombro o durante la marcha el miembro inferior que 
toma la carga y la pelvis ipsilateral ofrecen la estabilidad para el avance del miembro inferior opuesto.

La estabilidad se desarrolla en el niño en forma secuencial, en dirección proximal-distal ó céfalo-caudal, controlando primero la estabilidad estática y luego la dinámica (4): La estabilidad estática o mecánica se da por cocontracción de los músculos del plano frontal y sagital del segmento en juego, donde todavía no se controla grupos musculares antagonistas, por tanto, no hay desplazamiento del segmento y se muestra en fijación fisiológica.

La estabilidad dinámica resulta de la coordinación del movimiento desarrollada gracias al juego de inhibición recíproca de los músculos en un segmento que ya ha pasterizado la estabilidad mecánica y en donde se logra balancear la actividad de los grupos musculares antagonistas, por lo tanto se permite el desplazamiento.
Aunque cada segmento corporal lo hace en forma similar, miremos sólo el caso de las cinturas escapular y pélvica: cerca al cuarto mes el niño muestra estabilidad mecánica en la cintura escapular lo cual se ve porque el niño es capaz de mantenerse en prono sobre antebrazos, en este momento no hay movimiento, solo sostiene la posición, todavia no puede trasladar carga a un hombro para que el opuesto haga un alcance. En el sexto mes la cintura pélvica empieza su entrenamiento en la estabilidad estática y le sirve de punto de estabilidad a la escápula para que sea dinámica y pueda hacer alcances, más tarde la pelvis será dinámica en la medida en que rodillas y cuello de pie le den la estabilidad. Esta secuencia de eventos puede observarse cuando se revisa todo el desarrollo motriz por edades y por segmentos.

\section{El control postural y la importancia de la simetria}

La cintura escapular, está oficialmente constituida por el humero, la escápula, la clavícula y el esternón. Sin embargo, dada la extensión quinesiológica involucrada y las relaciones del movimiento entre la cintura escapular $y$ el movimiento corporal general, se consideran parte esencial del control dinámico de la cintura escapular: la caja costal, la espina, la cabeza, y la pelvis. Las piezas del complejo del hombro se mueven rítmicamente y en sincronía con cada otra y con el resto del cuerpo (5).

La cintura escapular es un sistema flotante con muchas partes que se interrelacionan. El brazo, la escápula y la clavícula se unen en la parte anterior del cuerpo por la articulación esternoclavicular. La fosa glenoidal es pequeña y superficial en comparación a la gran cabeza humeral, permitiendo amplios rangos del movimiento en el 
brazo, con poca estabilidad articular. El esternón, entonces, es la única parte del complejo del hombro que tiene buen soporte biomecánico (6). Por tanto, este sistema no tiene buen soporte biomecánico para su estabilidad, pero, delega el control de su estructura para los alcances y el traslado en el balance de la actividad muscular.

El control postural necesita del trabajo simétrico de flexión y extensión para neutralizar la acción de la gravedad, se ve claramente en la posición sedente. Mantenerse en la línea media es el signo más evidente de este control y es el marco de referencia para todos los movimientos. Todo movimiento eficiente trabaja desde este eje central que es la línea media. "EI manejo de la línea media permite el control de la postura y el movimiento, mientras usamos las extremidades para funciones como vestido, alimentación, y extensión protectora" (7).

En la ausencia de este balance, el niño tiene que usar los miembros superiores para ayudar a controlar la postura, sacrificando así, la función de sus segmentos y la seguridad de la posición en que se encuentra. Cuando el control simétrico no está disponible, el cuerpo del niño busca y usa adaptaciones posturales (FIGURA 2).
El rango articular de la cintura escapular está limitado por los tejidos blandos y la inflexibilidad capsular en un niño recién nacido, como sucede con la espina y las demás extremidades. En consecuencia, se limitan los movimientos activos de las extremidades superiores de manera que el humero difícilmente se eleva por encima del nivel del hombro y no puede cruzar el tórax en aducción horizontal. Esto mismo se puede presentar de manera atípica en niños con desarrollo anormal o retraso debido a los cambios intrínsecos de los músculos afectados postural o neurológicamente.

Los movimientos al azar del recién nacido y más tarde los intentos de movimiento voluntario del bebé, alargan las estructuras del tejido blando y moviliza las cápsulas articulares de el esternón, la clavícula y el humero, facilitando el movimiento libre de la escápula sobre la reja costal. La posición de soporte de peso en prono activa los músculos del manguito rotador, creando estabilidad dentro de la articulación glenohumeral (8).

Similarmente, este concepto se puede aplicar en un niño mayor que por lesión neuromotora o retraso motor presente inestabilidad glenohumeral o falta de movilidad articular.

\section{Desarrollo del movimiento en la cintura escapular}

Al iniciar el desarrollo de la extensión de cabeza sobre cuello y la extensión torácica alta en posición prono, es necesario, que la carga del niño sea tras- 
ladada más posteriormente. El tórax por ende, se eleva ligeramente de la superficie de soporte, la carga se soporta sobre los brazos, llevando el húmero desde una abducción de $90^{\circ}$ en dirección de aducción horizontal con rotación externa y los codos terminan alineados en una vertical con los hombros. La escápula esta en mayor abducción y rotación superior con menor elevación e inclinación anterior (9). Estos cambios conllevan a mayor disociación escápulo humeral (el desarrollo de la estabilidad escapular sobre el tronco con la consecuente habilidad para realizar movimientos humerales aislados).

Alrededor del 6 mes de vida, los aductores de la escápula se usan por el niño para reforzar la extensión torácica cuando quiere elevar su mirada y/o sentarse erguido. Esto ocurre como refuerzo a la extensión en posiciones antigravedad pero limita los alcances y manipulaciones porque así la escápula es incapaz de estabilizar el movimiento humeral (FIGURA 2).

Semanas mas tarde, iniciado ya el séptimo mes, el niño en posición prono presenta la reacción de Landau donde muestra mayor libertad de movimientos en forma de nadado porque los músculos espinales mantienen mayor extensión paravertebral (tronco superior). La escápula esta libre y puede proveer estabilidad para que el humero se desplace. Así, los codos se extienden para alcanzar. La actividad de extensión y el alcance unilateral llevan al desarrollo de traslado de carga, elongación lateral de tronco y flexión del tronco contralateral (FIGURA 1). Estas reacciones de endereza- miento se ven en uso más adelante durante las actividades de juego en prono (10).

En la medida en que se desarrolla un mejor control del hombro, el niño puede además apoyar sobre brazos semiextendidos (FIGURA3) con flexión de hombro mayor de $90^{\circ}$. Esta posición continúa facilitando la disociación escápulo humeral. Además, en supino al levantar cabeza y extremidades inferiores para agarrarse los pies, el niño refuerza fisiológicamente el estiramiento sobre el dorsal ancho y aumenta el rango de la extensión espinal. Esto ayuda a inhibir la fuerte actividad extensora y da mayor estiramiento a su musculatura.

Durante el 6-7 mes, aunque la fuerza de actividades flexor /extensor esta balanceada en prono y supino, durante las posiciones erguidas no sucede lo mismo. En sedente ef niño se inclina adelante y se apoya en brazos extendidos (FIGURA 3). Si se le da apoyo en sedente, la extensión de tronco superior es suficiente para permitir el alcance anterior con las extremidades superiores, no siendo igual en bípedo con apoyo donde la adducción escapular se usa para reforzar la extensión espinal. Esto muestra falta de maduración en la extensión toracolumbar durante para la posición sedente y uso de aductores de escápula para reforzar la extensión torácica durante la bipedestación (FIGURA 4).

La posición del hombro en el bebe, en relación con su cadera, en posición antigravedad (como en sedente) es una indicación del desarrollo y el control de la musculatura del tronco (11). 
De esta manera cuando los extensores están mas desarrollados que los flexores, los hombros estarán en una línea vertical mas delante de las caderas. El control flexor debe desarrollarse y se facilita si el bebé mantiene la posición de los hombros detrás de las caderas. Entonces el balance de la fuerza y control entre flexores y extensores permitirá al niño sentarse en posición erecta con hombros y caderas en alineamiento vertical. (FIGURA 3).

También, en la medida que el bebé se apoya sobre sus brazos extendidos con 90 grados de flexión de hombro se de- sarrolla una mejor integración y control de la reacción protectiva hacia delante (12). En prono el control del hombro conlleva al traslado de peso en esta posición al rotar externamente el hombro sobre el humero. Esta maduración del control de hombro además le permite al bebé empujar hacia atrás mientras esta en prono. (FIGURA 5).

Este aumento de los traslados de peso en sentido lateral y antero-posterior de los miembros superiores más adelante facilita el desarrollo de las rotaciones de cintura escapular y la disociación de la extremidad superior.

\section{Actividad motora y control postural}

Durante el proceso de maduración del control en la cintura escapular se origina simultáneamente cambios en la actividad motora de estructuras relacionadas y adquisición del control de nuevas posturas. A continuación podemos revisar las funciones de la cintura escapular en el control de movimientos y posturas en diferentes estructuras y posiciones.

\section{En la extremidad superior}

La adquisición de habilidades motrices en ef miembro superior facilita el aprendizaje de la motricidad fina, este desarrollo se da a partir de la estabilidad biomecánica y dinámica que le ofrece la estructura escapular.

En la posición prono, el niño experimenta información propioceptiva y ki- nestésica importante a través de todos los segmentos de la cintura escapular y las extremidades superiores. Experiencias cruciales se reciben en esta posición donde los brazos soportan y mueven dinámicamente el peso del cuerpo. Además, los miembros superiores tienen gran influencia en el desarrollo de las costillas, la espina dorsal, la pelvis y los miembros inferiores. En forma recíproca, la información que las extremidades superiores reciben depende en la secuencia del desarrollo que toma lugar en el resto del cuerpo. Es el caso del niño de 3 a 5 meses cuando transfiere el peso de su cuerpo desde los hombros hacia el abdomen, pelvis y fémur. Esto crea el potencial para el desarrollo del control abdominal. En forma revertida, la actividad abdominal provee estabilidad de la cintura escapular sobre el tronco, 
haciendo posible el control dinámico de los miembros superiores para el alcance y el agarre. La interrelación entre el desarrollo motor de la motricidad gruesa y la motricidad fina es increíble e indivisible (13).

La fuerza en flexión y extensión del codo incrementa simultáneamente con el desarrollo del apoyo sobre miembros superiores en extensión. En el apoyo sobre antebrazos, el niño traslada carga mediante la rotación externa sobre su brazo, causando supinación del antebrazo y soporte de carga sobre el borde cubital de la mano que soporta carga. El inicio de la supinación ocurre en prono y en los alcances en supino. Para desarrollar supinación dentro de las actividades motoras de un niño con desarrollo normal o anormal, es necesario tener en cuenta la forma cómo se inicia. (FIGURA 5).

El soporte y el traslado de carga sobre los miembros superiores en extensión de codos, ocurre en prono con traslado de carga primariamente sobre el borde cubital de la mano. En la medida en que el control lateral del tronco continúa desarrollándose, el niño puede además trasladar carga y hacer alcances sobre miembros superiores en extensión. El niño hace alcances en supino con codos totalmente extendidos y antebrazos en supinación. La extensión de codo controlada y resistida se demuestra en la reacción protectora hacia delante y en el empuje hacia atrás en prono. El niño asiste con miembros superiores durante todo el rango de tracción hacia sedente.

El pivoteo en prono (acción del movimiento de rebote que produce ascen- so y descenso repetido de sus partes) y el aumento en la disociación de extremidades superiores (una extremidad en patrón flexor y la otra extremidad en patrón extensor), permiten el desarrollo del alcance unilateral en posiciones sin soporte de carga (posición supina, decúbito lateral).

El soporte de carga sobre la superficie palmar y el traslado de carga desde el borde cubital al radial de la mano durante el pivoteo en prono desarrolla una mejor supinación y agarre de tipo radial. El mecido en posición cuadrúpeda es iniciado en los brazos. El niño usa la posición de sedente lateral para el juego y tracciona para ponerse de pie con los brazos (FIGURA 6). Transferencias de objetos mano a mano puede ocurrir en posiciones antigravedad en la medida que el control general del tronco mejora.

La maduración de los alcances ocurre principalmente con supinación completa de antebrazo incluso en posiciones antigravedad (prono, sedente, cuadrúpeda, rodillas y bípeda). El niño puede empujar objetos hacia delante $y$ atrás y de lado a lado en sedente, una vez ha desarrollado buen control de tronco. La diferenciación de las partes distales de las extremidades superiores desarrolla el uso aislado de los dedos para patrones de agarre refinados y liberación voluntaria.

\section{Control del tronco}

El desarrollo del control de tronco permite al niño usar la rotación cuando se traslada desde sedente en una banca y se baja a cuadrúpeda. En la medida que madura el control lateral de 
tronco el niño pasará de decúbito lateral a sentado lateral (FIGURA 6). El control lateral de tronco es además suficiente para permitir al niño cruzar y caminar usando solo una mano para apoyarse. Sin embargo, cuando la marcha independiente surge, asume una posición de alta guardia para reforzar la extensión axial con adducción y elevación escapular, extensión, adducción con rotación externa de hombros y flexión de codos (FIGURA 4). Cuando las reacciones de equilibrio se van desarrollando, el niño empieza a bajar sus brazos pero el humero permanece en adducción hasta su completo desarrollo en esta nueva forma de movilidad (la marcha).

\section{Reacciones posturales}

La reacción de extensión protectora hacia adelante se presenta en sedente con abdución escapular y buena extensión del tronco superior. La reacción de extensión protectora lateral, empieza cuando se desarrolla el control flexor en las posturas antigravedad.

Las reacciones protectoras laterales cuando están presentes en sedente, producen la posibilidad de aumentar el rango del decúbito lateral con el brazo apoyado en la mano y con extensión de codo. La extensión fuerte del codo resulta en una mejor reacción protectora lateral.

\section{Posicion de rodillas y bipeda}

Pensando en la acción reciproca de la que hablan Alexander, Boehme y Cupps, podemos ver otra situación que se revierte. Con la constante acción de halar hacia posiciones erguidas, como rodillas y bípeda, las extremidades superiores y la cintura escapular se vuelven más fuertes. A su vez, un mayor control en la cintura escapular, se manifiesta en mayor control del tronco durante la posición de rodillas y la bipedestación.

En una posición bípeda que necesite soporte de tronco inferior, hay signos de debilidad en el tronco superior, por lo tanto, el niño necesita apoyo lumbar y pélvico para iniciar movimientos de tipo rotacional. El aumento en la estabilidad del tronco superior le permite al niño jugar y alcanzar en bipeda cuando se le da soporte al tronco inferior y mucho mejor cuando activamente aprende a controlarlo.

El bebé tiene suficiente fuerza para halar de si mismo hacia bípedo y la flexión lateral del tronco superior provee la forma como inicialmente traslada el peso para cruzar entre los muebles. La estabilidad del tronco bien desarrollada permite al niño jugar en rodillas y su completo control le permite durante la marcha, liberar los miembros superiores para alcanzar, agarrar y acarrear objetos.

\section{Motricidad oral y respiracion}

Funcionalmente, el maxilar inferior tiene relación directa $e$ indirecta con el cráneo, la cara, el cuello y la cintura escapular mediante conexiones esqueléticas, musculares, nerviosas y faciales (14). Estas relaciones hacen pensar en la inherencia del control escápulo-humeral-clavícula-esternón sobre las actividades de la cavidad oral y el cuello, especialmente la alimentación $y$ la fonación. 
El marco óseo de la reja costal incluye las costillas, el esternón y la región torácica. Los mecanismos oral, faríngeo y respiratorio se modifican en la medida en que el bebé los usa funcionalmente. Estos mecanismos cambian significativamente en su contorno, forma, tamaño alineación y actividad muscular durante el desarrollo motor. La musculatura de la cintura escapular provee una fuente activa de estabilidad a la reja costal durante los movimientos y le lleva a cambiar de forma $y$ tamaño.

\section{Conclusiones}

Finalmente, puede decirse que la evaluación e intervención de patrones de movimiento debe acompañarse de una cuidadosa observación de la totalidad del niño y no de una única zona. El desarrollo de una habilidad motora no es un proceso aislado. Cada aspecto del control del movimiento procede de patrones gruesos para llegar a patrones de coordinación motora altamente refinados.

En el caso de la cintura escapular, se puede generar control motor en esta estructura para facilitar la actividad de otras partes del cuerpo. La cintura escapular es una estructura que ocupa un lugar èstratégico en el cuerpo, porque desde allí puede intervenir en el desarrollo de múltiples actividades
El control postural de la cabeza, el cuello, los hombros, el tronco superior afectan directamente la motricidad oral con sus funciones de fonación y alimentación. Un cuello es hiperextensión por falta de estabilidad escapular o por otra causa, dificulta la masticación y la deglución, la falta de control en el tronco superior retira la base de estabilidad para la reja costal, afecta negativamente el patrón respiratorio, y cambia su forma y tarnaño. El análisis de estos mecanismos sería tema para largos espacios de discusión y no compete profundizar en ellos en esta revisión.

como el desarrollo de habilidades motoras en el miembro superior, el control del tronco, reacciones posturales, los traslados desde y hacia diferentes posiciones, las habilidades motoras en sedente, rodillas y bípeda, y las funciones de alimentación, fonación y respiración.

En general, algunas posiciones de soporte de peso como prono sobre antebrazos, prono sobre manos, cuadrúpeda y sentado lateral, son las posiciones que proporcionan mayor control de la función del miembro superior y es la estructura escapular quien proporciona la estabilidad básica para que este control se inicie, se perfeccione y se mantenga. 


\section{Bibliografía}

(1) Levitt, Sophie BSC FT, MCSP. Tratamiento de la Parálisis Cerebral y del retraso motor. Madrid. 2000. Editorial Médica Panamericana. Pág. 39.

(2) Cupps, Barbara A. PJ. BS, NDTA. Developmental treatment: An interdisciplinary Approach. 1994.Lisle Illinois. Marianjoy Rehabilitation Hospital. Pág. 43.

(3) Cupps. Pág. 29.

(4) Girolami, Gay, PT., MS. Tratamiento del Neurodesarrollo para infantes con alteración cerebral motora. 1995. OHando. Regional Healthcare System. Pág. 24.

(5) Boehme, Regi, OTR. Improving Upper Body Control" 1988. Tucson, Arizona. Therapy Skill Builders. Pág. 20.

(6) Alexander, Riona Ph. D., CCC-SP, Boehme, Regi OTR, Cupps, Barbara. PT. Normal Development of Functional Motor Skills. Tucson, Arizona. 1993. Therapy skill Euilders. Pág 18.

\section{Figuras}

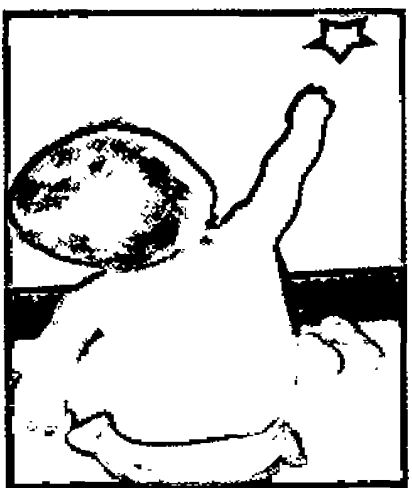

Figura 1. Alcance Unilateral en posición sedente: La estabilidad escapular da libertad al miembro superior para hacer alcances. El Tronco en el lado derecho elongado (soporta peso) y lado izquierdo del tronco acortado.
(7) Boehme, Regi. Pág. 70

(8) Boehme, Regi, OTR. "Approach to treatment of the baby" 1990. Tucson, Arizona. Therapy Skill Builders. Pág. 55.

(9) Boehme, Regi, 0TR. "Approach to treatment of the baby" 1990. Tucson, Arizona. Therapy Skill Builders. Pág. 25.

(10) Cupps. Pág. 35.

(11) Alexanderr. Pág. 99.

(12) Boehme, Regi، OTR. Improving Upper Body Control" 1988. Tucson, Arizona. Therapy Skill Builders. Păg. 81.

(13) Alexander, Rona. Pag. 72.

(14) Alexander, Rona. Pág. 24.

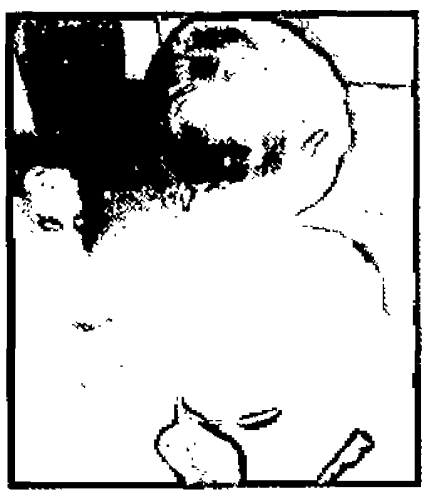

Figura 2. Sedente con adducción de escápula. Mantiene el miembro superior ocupado en dar estabilidad a la escápula para aumentar o mantener la extensión de tronco superior que todavia no es muy fuerte. 


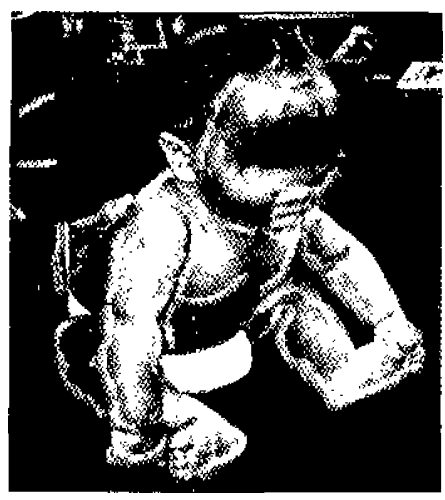

Figura 3. Sedente con apoyo de miembros superiores. Se observa extensión de tronco superior, con falta de extensión en tronco inferior que lo lleva a la inclinación anterior, en la línea perpendicular imaginaria los hombros están por delante de la pelvis.

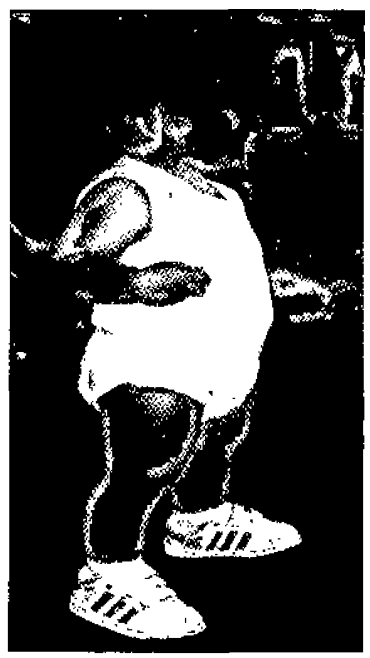

Figura 4. Posición bipeda con adducción escapular para aumentar extensión en tronco superior y estabilizarlo. El miembro superior se mantiene cerca al tronco con hombros en extensión. adducción y rotación interna y los codos en moderada flexión.

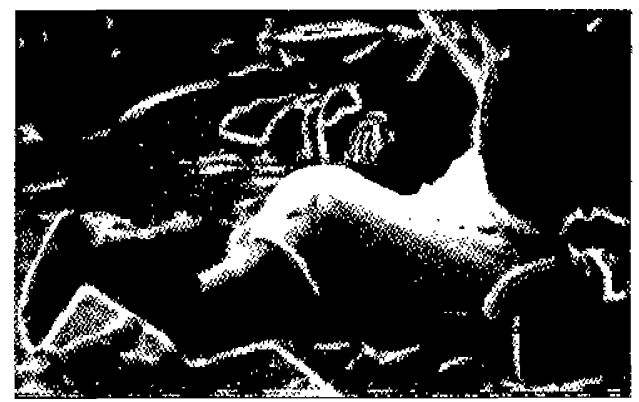

Figura 5. Posición prono. Alcance con miembro superior izquierdo y traslado de peso sobre miembro superior derecho que muestra rotación externa de hombro, antebrazo en supinación y apoyo sobre borde cubital de la mano.

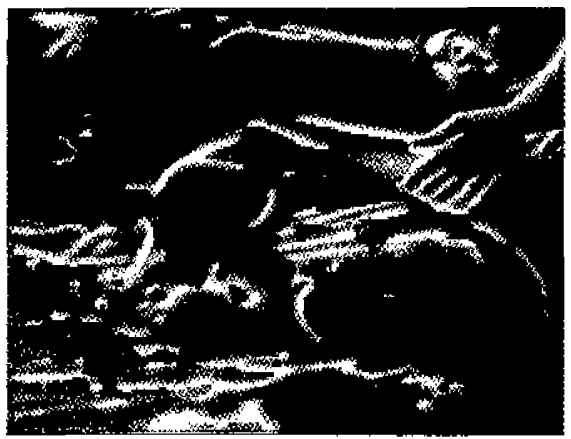

Figura 6. Sedente Lateral. Posición común para pasar de una posición a otra mientras en niño se mueve en el suelo. Facilita el alcancé de objetos y desarrolla rotaciones y control lateral de troncin 